\title{
Tratamiento de la colecistocoledocolitiasis en un solo tiempo con la técnica de rendez-vous. Hospital Nacional de Itaugua
}

\author{
Management of Cholecystocholedocholithiasis in a single stage with the \\ rendez-vous procedure. Hospital Nacional de Itauguá
}

\author{
${ }^{\star}$ Carlos Rene Adorno Garayo ${ }^{1},{ }^{*}$ Ruth Elizabeth Cabrera Maciel ${ }^{2,},{ }^{\star * *}$ José Manuel González \\ Vazquez $^{2},{ }^{* * * *}$ Miguel Ferreira Bogado ${ }^{2}$
}

1. Hospital Nacional de Itauguá, Servicio de Endoscopia Digestiva. Paraguay

2. Hospital Nacional de Itauguá, Servicio de Cirugía general. Paraguay

\section{RESUMEN}

Introducción: Describimos nuestra experiencia inicial con la utilización del método laparoendoscópico en un solo tiempo "RENDEZ-VOUS" en el manejo de los pacientes con litiasis vesicular y sospecha de coledocolitiasis. Objetivo: Describir la tasa de éxito, complicaciones, estancia hospitalaria con el método Rendez vous. Metodología: Se aplicó el método de Rendez-vous en 12 pacientes que ingresaron con los diagnósticos de litiasis vesicular y sospecha de coledocolitiasis en forma seleccionada de un muestreo no probabilístico de casos consecutivos con una muestra de 25 pacientes que cumplieron los criterios de inclusión. Resultado: Se realizó la Colangiografía intraoperatoria en 25 pacientes, se confirmó la presencia de coledocolitiasis en 14 pacientes (56 \%) y se logró la extracción exitosa de cálculos de la vía biliar en 12 pacientes (48 \%) a través de la colangiografía retrógrada endoscópica selectiva sobre guía con loop para Rendez-vous por vía trancística. No se reportaron casos de pancreatitis post procedimientos ni casos de litiasis coledociana residual en 6 meses de seguimiento. La media de estadía hospitalaria fue de 5,8 días y de estadía pos operatoria de 1,2 días. Conclusión: El método de Rendez-vous se presenta como una alternativa terapéutica mínima invasiva a los demás tipos de tratamientos para la litiasis coledociana.

Palabras clave: Rendez-vous, Coledocolitiasis, litiasis vesicular, Colangiografía retrógrada endoscópica, litiasis de la vía biliar.

\section{ASTRACT}

We describe our initial experience with the use of the single-stage laparoendoscopic method "RENDEZ-VOUS" in the management of patients with gallstones and suspected choledocholithiasis. Objective: To describe the success rate, complications, and hospital stay with the Rendez vous method. Methodology: The Rendez-vous method was applied in 12 patients who were admitted with the diagnoses of gallstones and suspected choledocholithiasis, selected from a non-probabilistic sampling of consecutive cases with a sample of 25 patients who met the inclusion criteria. Result: Intraoperative cholangiography was performed in 25 patients, the presence of choledocholithiasis was confirmed in 14 patients (56\%), and the successful removal of bile duct stones was achieved in 12 patients (48\%) through endoscopic retrograde cholangiography. selective on guide with loop for Rendez-vous by transcystic way. No cases of post-procedure pancreatitis or cases of residual choledochal lithiasis were reported in 6 months of follow-up. The mean hospital stay was 5.8 days and the postoperative stay was 1.2 days. Conclusion: The Rendez-vous method is presented as a minimally invasive therapeutic alternative to other types of treatments for choledochal lithiasis.

Keywords: Rendez-vous, Choledocholithiasis, gallstones, Endoscopic retrograde cholangiography, bile duct lithiasis.

\section{INTRODUCCIÓN}

El cálculo del conducto biliar común ocurre en alrededor del 3 $\%$ al $15 \%$ de los pacientes sometidos a colecistectomia laparoscopica, en la era de la colecistectomia abierta el aclaramiento endoscopio pre operatorio del conducto biliar común seguido de una colecistectomia abierta fue comparable o incluso se consideró inferior a la colecistectomia en una sola etapa y la coledocolitotomia laparoscópica. No hay un consenso sobre la estrategia de gestión ideal para este tipo de pacientes, tradicionalmente, se le ofreció la cirugía abierta, pero con el advenimiento de la colangiografía retrógrada endoscópica CPRE, y la colecistectomía laparoscópica CL abordajes mínimo invasivos casi se había sustituido la laparotomía debido a las ventajas de

* Jefe del Servicio de Endoscopia Digestiva del Hospital Nacional de Itauguá.

** Residente de tercer año del Servicio de Cirugia general del Hospital Nacional de Itauguá.

*** Especialista en Cirugía General, Hospital Regional de Villarrica.

**** Jefe de sala del Servicio de Cirugia General del Hospital Nacional de Itaugua.

Autor Correspondiente: Dra. Ruth Elizabeth Cabrera Maciel. Ministerio de Salud Pública y Bienestar Social, Centro Médico Nacional, Hospital Nacional, Departamento de Cirugía. Itauguá, Paraguay - Correo electrónico: ruth9.cabrera@gmail.com

Recibido: 27/07/2020 - Aceptado: 21/10/2020

Este es un artículo publicado en acceso abierto bajo una licencia Creative Commons 
estos procedimientos, estos procedimientos se han convertido en alternativas viables para la exploración y solución del cálculo del conducto biliar común ${ }^{1}$.

Muchos de los investigadores alrededor del mundo se han planteado la meta de lograr la solución de ambas patologías con el menor costo, mayor tasa de éxito y menor número de complicaciones posibles, se ha encontrado que el abordaje endoscópico es similar a la exploración laparoscópica del cálculo de la vía biliar común en términos de limpieza exitosa de los cálculos, como morbilidad y mortalidad excepto por una estancia hospitalaria más prolongada ${ }^{1}$.

El manejo más frecuente de la litiasis vesicular con coledocolitiasis en el Hospital Nacional de Itauguá de la realización de colangiopancreatografía retrógrada endoscópica (CPRE) preoperatoria seguida de colecistectomía laparoscópica (CL) 24 a 48 horas después como en la mayoría de los centros de referencia. Hemos iniciado también en nuestro hospital la opción de realizar el tratamiento aplicando la exploración laparoscópica de las vías biliares (ELVB), con nuestras limitaciones en infraestructura y la curva de aprendizaje que vamos adquiriendo en nuestro servicio quirúrgico y endoscópico.

Es importante destacar que en diferentes publicaciones existe un $35 \%$ a $60 \%$ de los pacientes con alta sospecha de coledocolitiasis a los que se les practica CPRE, esta resulta negativa para coledocolitiasis ${ }^{(1,2,3,4)}$. Más aun, la CPRE que es un buen método efectivo para el diagnóstico y tratamiento de la coledocolitiasis, tiene asociado una morbilidad de unos 3\% a 9\% según distintas series en forma global $^{(2,3,4,5)}$. Por esa razón se justifica plenamente realizar un procedimiento diagnóstico y terapéutico que tenga como finalidad resolver el problema y evitar el daño iatrogénico que puede causar la CPRE y en un solo tiempo. Teniendo en cuenta estos requisitos contamos en nuestro servicio con el método laparo-endoscópico Rendez-vous, el cual evita los tres elementos de riesgo de la CPRE, la inyección retrógrada de contraste al Wirsung, la canulación del Wirsung, la manipulación prolongada de la papila ${ }^{6,7}$. La mayoría de los estudios han demostrado que las estrategias de dos sesiones es eficiente y segura, sin embargo este enfoque tiene inconvenientes resultados negativos altos; ( $40 \%$ al $70 \%$ ) que exponen a los pacientes en una intervención endoscópica innecesaria de riesgo ${ }^{(1)}$.

En el servicio de Cirugía y Endoscopia del Hospital Nacional de Itauguá el método de Rendez-vous se nos presenta como una alternativa útil como resolución mínimamente invasiva y en un solo tiempo de la coledocolitiasis concomitante con la litiasis vesicular. En este trabajo utilizamos el método por acceso endoscópico controlado a la vía biliar sobre una guía hidrofílica tipo loop colocada previamente por vía trancística durante la colecistectomía laparoscópica y describimos los resultados obtenidos.

\section{MATERIAL Y MÉTODO}

Todos los pacientes con diagnóstico de litiasis vesicular y probabilidad de coledocolitiasis manejados en el Servicio de Cirugía General y Endoscopía Digestiva de julio 2013 a julio 2016 realizando un estudio de tipo prospectivo, descriptivo. La población está compuesta por todos aquellos pacientes mayores de 15 años que ingresaron al servicio de urgencias del Hospital Nacional de Itauguá con los diagnósticos de litiasis vesicular y Sospecha Intermedia de coledocolitiasis según protocolo de $\mathrm{ASGE}^{(10)}$. La muestra está conformada por 25 pacientes con los diagnósticos de litiasis vesicular y sospecha intermedia de coledocolitiasis que cumplieron los criterios de inclusión y exclusión.

Tabla 1. Criterios de inclusión.

1. Pacientes de cualquier género

2. Pacientes mayores de 15 años

3. Pacientes ASA I y II.

4. Diagnóstico de litiasis vesicular por ultrasonido abdominal.

5. Bilirrubina de $1.7 \mathrm{mg} / \mathrm{dl}$ a $4 \mathrm{mg} / \mathrm{dl}$.

6. Sospecha de Coledocolitiasis riesgo intermedio por los Criterios de ASGE.

Tabla 2. Criterios de exclusión.

1. Pacientes ASA III y IV.

2. CPRE con esfinterotomía previa.

3. Colangitis.

4. Obstrucción duodenal.

5. Coagulopatías.

6. Cirugía abdominal previa.

7. Embarazo durante el ingreso

8. Pancreatitis Aguda.

9. Evidencia o Sospecha de patología neoplásica peri ampular.

\section{Descripción de la Técnica}

El equipo médico multidisciplinario está conformado por el equipo quirúrgico (cirujano endoscopista, dos ayudantes, enfermera instrumentista y circulante), equipo de Anestesiología, equipo de endoscopia (enfermera ayudante) y un técnico radiólogo (Figura 1).

\section{Método de Rendez-vous. Paso a paso}

Tiempo laparoscópico: Anestesia general, previas medidas de asepsia y antisepsia, colocación de campos estériles, técnica abierta para introducción de trocares de $10 \mathrm{~mm}$ en número de dos y de $5 \mathrm{~mm}$ número de dos. Óptica de 30 grados. Colecistectomía propiamente dicha, disección del triángulo de Calot, identificación del conducto cístico y la arteria cística, clipado con LT 400 de la arteria, previa a la sección del conducto cístico se realiza el destechamiento casi completo del cuerpo y fondo vesicular de manera a dejar colgado nada menos que por un pequeño colgajo para facilitar la colecistectomía una vez termine el procedimiento de CPRE si se da lugar. Sección parcial del conducto cístico y colocación de un 5to trocar de $3 \mathrm{~mm}$ para paso de sonda o catéter para colangiografía intra operatoria (CPO). Se utiliza previa al momento de la contrastación $20 \mathrm{mg}$ de Bromuro de hioscina endovenosa. Se realiza la colangiografía trancística con la instilación de contraste hidrosoluble bajo visión directa con fluoroscopia de Arco en C. Siemens. El siguiente paso va depender de lo que se halla durante la CPO, en caso de no evidenciar coledocolitiasis se realiza el clipado del conducto cístico la sección total y se completa la colecistectomía, si se confirma la presencia de coledocolitiasis se continua el procedimiento con la técnica de Rendez-vous (Figura 2) y, colocación del Catéter Trancístico.

Tiempo endoscópico: si se realizó el diagnóstico de coledocolitiasis se procede a la colocación de una guía hidrofilica de 0,0035 con loop especial a través del conducto cístico (Figura 3). Y se comprueba su paso al duodeno por fluoroscopia.

En este momento el endoscopista se encarga de la introducción del Duodenoscopio en posición supina, se procede a 
visualizar la guía hidrofílica y el loop del mismo, se realiza la cateterización del ojal de la guía con guía hidrofílica normal que se encuentra en el Papilotomo (Figura 4).

Una vez realizada la introducción de la guía hidrofílica normal dentro de la guía con ojal que fue puesta a través del conducto cístico, eso nos permite la cateterización directa de la vía biliar gracias a la extracción que realiza el ayudante desde la guía puesta en el cístico. Eso nos permite entrar directamente sin manosear la papila y a sabiendas de que existe litiasis coledociana gracias a la CPO hecha previamente, se realiza la papilotomía endoscópica y a partir de ese momento se procede como una CPRE común y corriente, se utilizan la canastilla de Dormia o

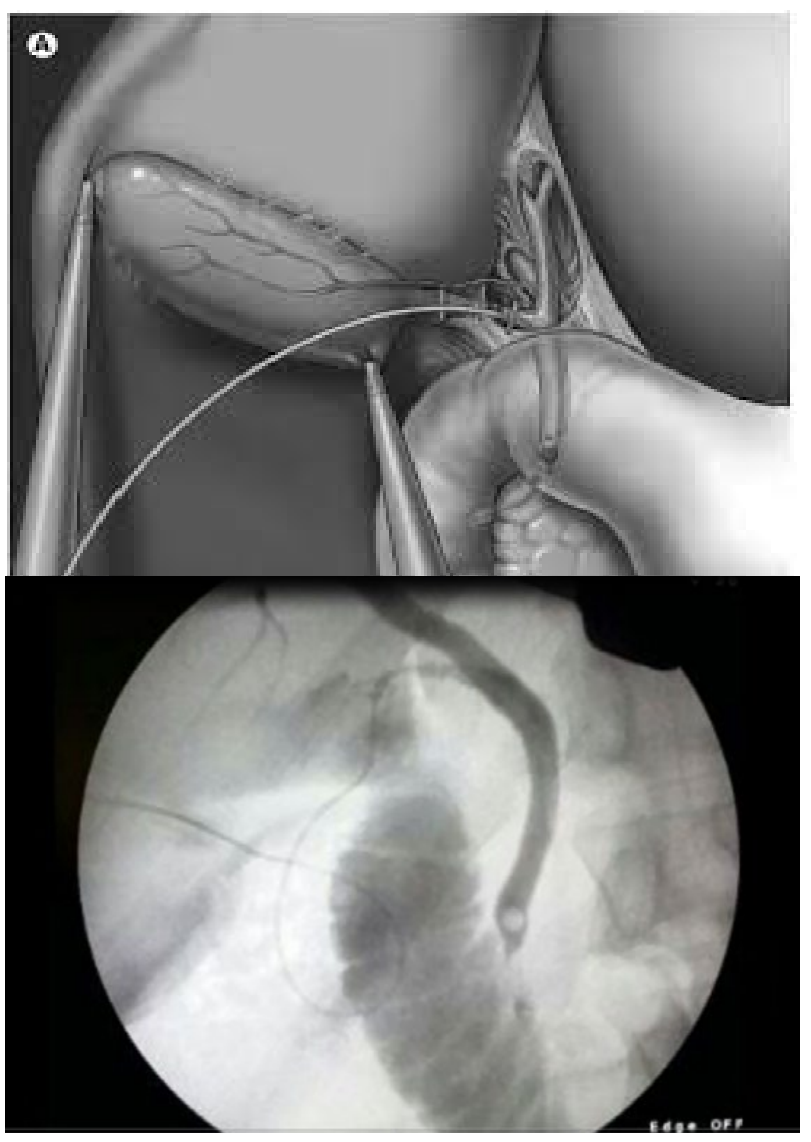

Figura 1. Catéter trancístico.

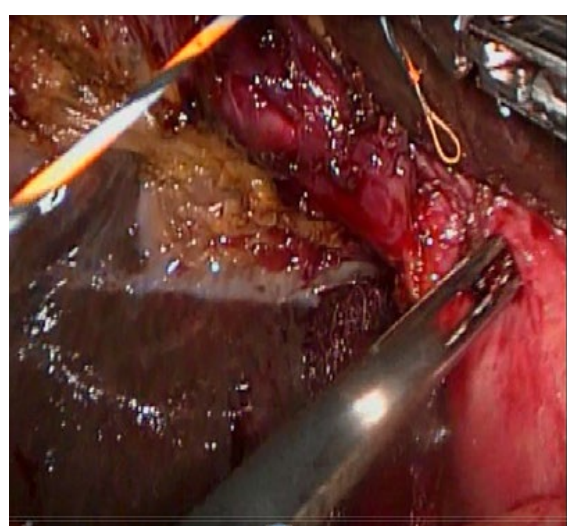

Figura 2. Paso de guía hidrofilica con loop. el Balón extractor para poder realizar la extracción del cálculo coledociano (Figura 5 y Figura 6).

Segundo tiempo laparoscópico: se completa la colecistectomía. Se extrae la guía hidrofílica y se realiza el clipado del conducto cístico así como la sección completa. Se completa la colecistectomía por técnica habitual.

\section{RESULTADOS}

Este estudio está conformado por 25 pacientes, 21 pacientes del sexo femenino y 4 pacientes del sexo masculino, con edad promedio de 38,3 (17-74 años) que acudieron a urgencias y pasaron a sala de internados del Hospital Nacional de Itauguá, con los

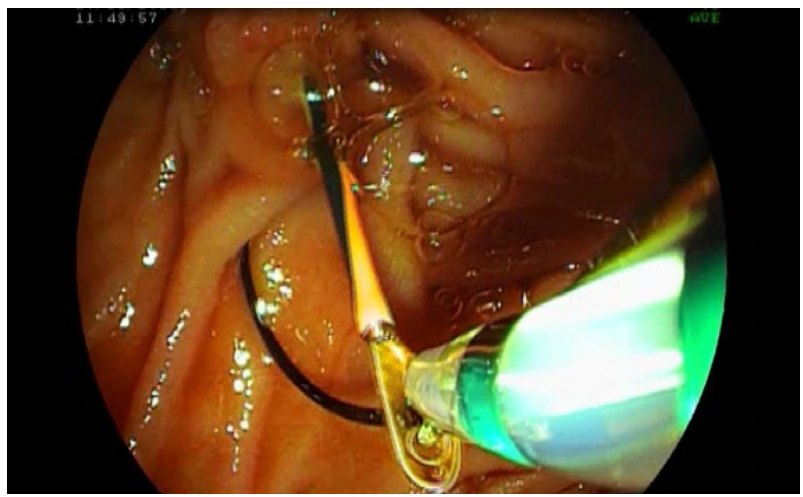

Figura 3. Cateterización de la vía biliar, a través de la guía hidrofílica con ojal.

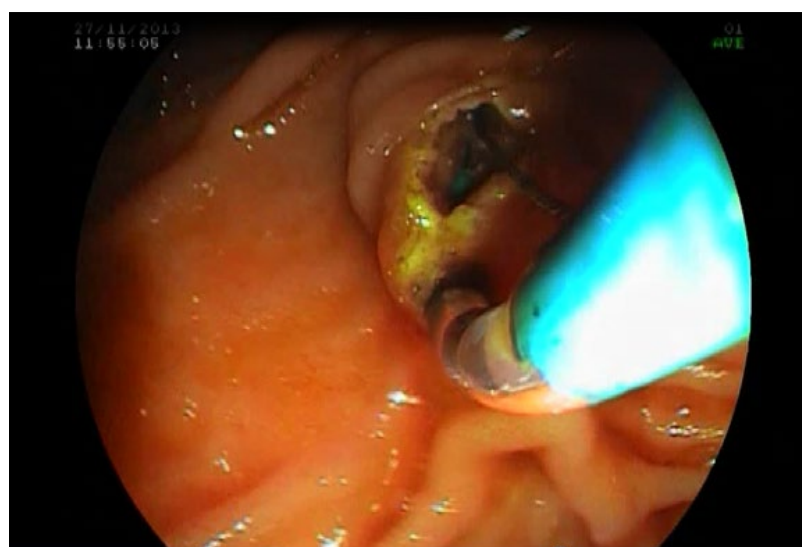

Figura 4. Papilotomía.

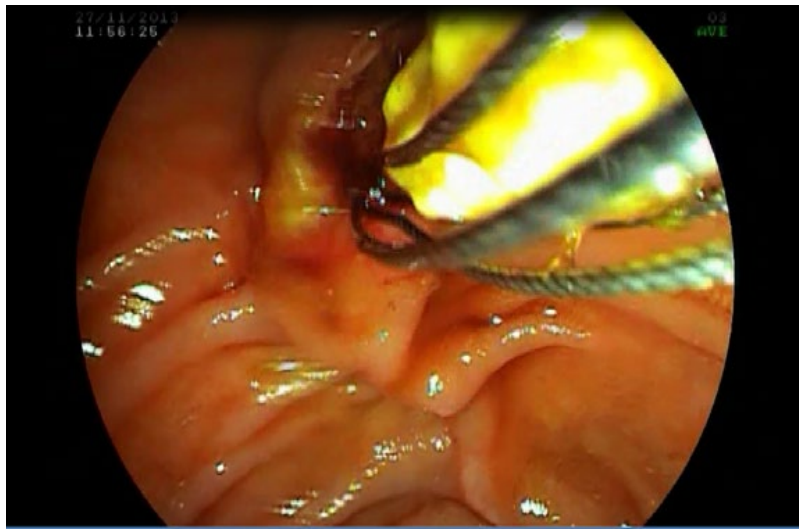

Figura 5. Extracción del cálculo. 
diagnósticos de litiasis vesicular y sospecha intermedia de coledocolitiasis desde 2013 hasta 2016.

Tabla 3. Características de los pacientes al momento del ingreso.

\begin{tabular}{lccc}
\hline & HOMBRES & MUJERES & PROMEDIO \\
Sexo & 4 & 21 & \\
Edad & 65 & 33,2 & 38,3 \\
IMC ${ }^{(\mathrm{kg} / \mathrm{m} 2)}$ & 24,25 & 25,1 & 25,04 \\
Ictericia & $71 \%$ & $75 \%$ & $72 \%$ \\
BT ${ }^{(\mathrm{mg} / \mathrm{d})}$ & 3,47 & 3,59 & 3,57 \\
CV.VBP US & $0 \%$ & $33 \%$ & $28 \%$ \\
\hline
\end{tabular}

CV.VBP US (calculo visible en vía biliar principal por ecografía abdominal).

El diagnóstico de litiasis vesicular se realizó por ecografía abdominal en todos los casos. Para el diagnóstico o sospecha intermedia de litiasis vesicular se tomaron como criterios los resumidos en la Tabla 4, según protocolo de ASGE ${ }^{10}$.

Tabla 4. Criterios de Diagnostico para sospecha de coledocolitiasis ${ }^{(10)}$.

\section{PREDICTORES DE COLEDOCOLITIASIS}

\section{Muy Fuertes}

Calculo en VBP por Ecografía

Colangitis Clínica

Bilirrubina mayor a $4 \mathrm{mg} / \mathrm{dl}$

\section{Fuertes}

VBP dilatada por ecografía (mayor a $6 \mathrm{~mm}$ en vesícula in situ)

Bilirrubina entre 1.8 a $4 \mathrm{mg} / \mathrm{dl}$

\section{Moderado}

Hepatograma alterado además de la Bilirrubina

Mayor a 55 años

Pancreatitis Aguda Litiasico

\section{$>$ Presencia de Cualquier predictor muy fuerte Alto \\ $>$ Presencia de 2 predictores Fuertes \\ Alto \\ $>$ Sin Predictores \\ Bajo \\ $>$ Todos los demás pacientes Intermedio}

En 25 pacientes se realizó la colangiografía intraoperatoria como paso inicial, se confirmó el diagnóstico de coledocolitiasis en 14 pacientes (56\%), en los 11 pacientes (44\%) cuyo CIO fue negativo se realizó colecistectomía laparoscópica sin complicaciones postoperatorias ni ictericia recurrente en 6 meses de seguimiento.

En 12 pacientes cuya CIO resultó positiva se procedió a realizar la canulación selectiva de la vía biliar principal y colocación de la guía hidrofílica con ojal con paso hasta el duodeno de forma exitosa. En estos 12 casos se logra la extracción completa del cálculo y/o cálculos de la vía biliar (85.7\%). En dos (14,2\%) pacientes cuya CIO fueron positivas, en el 1ro no se logra pasar la guía debido a la presencia de 4 cálculos de gran tamaño que no permiten el paso, se decidió realizar una CPRE intra operatoria común, pero el hallazgo endoscópico nos demostró la pre- sencia de una papila diverticular que prácticamente nos impidió seguir con el procedimiento, se decide realizar la extracción de la misma por vía totalmente laparoscópica con drenaje de kher sin complicaciones. En un 2do paciente tuvimos el mismo problema, el cálculo enclavado en colédoco distal no nos permite el paso de la guía además de medir aproximadamente $2 \mathrm{~cm}$ por lo que se decide la cirugía convencional, coledocotomía y extracción del cálculo y posterior drenaje de kher. Nuestra tasa de Conversión a laparotomía fue del $(7,1 \%) 1$ solo paciente y totalmente laparoscópica del (7,1\%) 1 solo paciente. Tabla 5.

Tabla 5. Hallazgos Operatorios.

\begin{tabular}{lccc}
\hline & HOMBRES & MUJERES & TOTAL \\
1. ClO positiva & 2 & 12 & 14 \\
2. CaVBP & 1 & 11 & 12 \\
3. RV completo & 1 & 11 & 12 \\
\hline
\end{tabular}

CIO: colangiografia intra operatoria.

CaVBP: canulación selectiva de la vía biliar principal efectiva.

$R V$ : procedimientos completados por método de Rendez-vous laparoendoscópicos.

El tiempo de estadía hospitalaria promedio para nuestros pacientes fue de 5,8 días (2-23) y de estadía pos operatorio de 1,2 días (1-8 días). En los controles laboratoriales posterior al procedimiento no se evidenciaron hiperamilasemia a las $24 \mathrm{hs}$. No hubo casos de mortalidad, tuvimos una infección de herida operatoria en el paciente que fue sometido a colecistectomía convencional más coledocotomía y drenaje de kher. Tabla 6.

\section{DISCUSIÓN}

En este trabajo demostramos los resultados obtenidos con el método de Rendez-vous para la resolución de dos patologias en un solo tiempo en nuestros pacientes con diagnóstico de litiasis vesicular y sospecha intermedia de litiasis coledociana. El tratamiento secuencial en dos tiempos (CPRE con CL) o en un solo tiempo (Rendez-vous o ELVB) pueden ser factibles de forma segura para el manejo de estos pacientes, siendo las únicas dificultades para la aplicación entre uno u otro método la experiencia del equipo humano encargado, así como la disponibilidad de los recursos en infraestructura. El tratamiento en un solo tiempo permite evitar una segunda anestesia, evitar las CPRES negativas y por ende sus complicaciones ${ }^{(1,2,3,4,5,6)}$.

Otra opción que contamos en nuestro servicio como tratamiento en un solo tiempo mínimamente invasivo es la Exploración laparoscópica de la Vía biliar, ya sea esta por abordaje trancístico o coledocotomía laparoscópica, pero es importante destacar que depende mucho de la experiencia del cirujano y la curva de aprendizaje con respecto a este procedimiento además de los instrumentales especiales que se utilizan en dicho procedimiento ${ }^{(8,9)}$. El abordaje trancístico fue efectivo en $44,5 \%$ aproximadamente en distintas series, siendo su mayor limitante la presencia de cálculos múltiples, mayores a $8 \mathrm{~mm}$, intrahepáticos y los cálculos enclavados. En cuanto a la coledocotomia se sabe que es una técnica compleja limitada a expertos en el área ${ }^{(7,8)}$.

Nuestra técnica de Rendez-vous o colangiopancreatografía intra operatoria es fifícil técnicamente debido a la posición supina del paciente, pero con la ayuda de las diferentes técnicas de encuentro (con canastilla de Dormia, guía común, catéter trancístico con pre corte por encima y la guía para Rendez-vous 
Tabla 6. Días de hospitalización total y de egreso post operatorio por paciente según procedimiento realizado y diagnóstico final.

\begin{tabular}{|c|c|c|c|c|}
\hline Paciente & $\begin{array}{c}\text { Días } \\
\text { Hospital } \\
\text { total }\end{array}$ & $\begin{array}{l}\text { Días de } \\
\text { Egreso } \\
\text { pos op }\end{array}$ & $\begin{array}{l}\text { Procedimiento } \\
\text { realizado. }\end{array}$ & Diagnóstico final. \\
\hline 1 & 7 & 1 & RV & LV-CDL \\
\hline 2 & 4 & 1 & RV & LV-CDL \\
\hline 3 & 8 & 1 & RV & LV-CDL \\
\hline 4 & 7 & 1 & CIO-CVL & LV \\
\hline 5 & 9 & 1 & CIO-CVL & LV \\
\hline 6 & 8 & 1 & RV & LV-CDL \\
\hline 7 & 3 & 1 & RV & LV-CDL \\
\hline 8 & 2 & 1 & RV & LV-CDL \\
\hline 9 & 12 & 8 & COLEDOCOTOMIA & $\begin{array}{l}\text { LV-CDL } \\
\text { LAPAROSCOPICA }\end{array}$ \\
\hline 10 & 4 & 1 & CIO-CVL & LV \\
\hline 11 & 2 & 1 & RV & LV-CDL \\
\hline 12 & 7 & 7 & $\begin{array}{l}\text { COLEDOCOTOMIA } \\
\text { KEHR }\end{array}$ & $\begin{array}{l}\text { LV-CDL } \\
\text { ABIERTA. }\end{array}$ \\
\hline 13 & 6 & 1 & RV & LV-CDL \\
\hline 14 & 3 & 1 & CIO-CVL & LV \\
\hline 15 & 4 & 1 & CIO-CVL & LV \\
\hline 16 & 23 & 1 & CIO-CVL & LV \\
\hline 17 & 2 & 1 & CIO-CVL & LV \\
\hline 18 & 2 & 1 & ClO-CVL & LV \\
\hline 19 & 2 & 1 & CIO-CVL & LV \\
\hline 20 & 3 & 1 & CIO-CVL & LV \\
\hline 21 & 2 & 1 & RV & LV-CDL \\
\hline 22 & 2 & 1 & CIO-CVL & LV \\
\hline 23 & 4 & 1 & $\mathrm{RV}$ & LV-CDL \\
\hline 24 & 4 & 1 & RV & LV-CDL \\
\hline 25 & 5 & 1 & RV & LV-CDL \\
\hline
\end{tabular}

Cio: colangiografía intraoperatoria LV: Litiasis Vesicular CDL: Coledocolitiasis. VBP: vía biliar principal, RV (procedimientos completados por Rendez-vous). con ojal), se hace menos dificultosa y con seguridad se puede ingresar a la vía biliar independientemente de la anatomía papilar. Además, se realiza el procedimiento ya a sabiendas de la presencia de un cálculo, sin la necesidad de inyectar contraste en forma retrógrada y con la seguridad de no manosear la papila ni ingresar al conducto pancreático ${ }^{(1,2,3,4)}$.

Un estudio comparativo no aleatorio reportó mayor facilidad de la técnica utilizando el Rendez-vous y una reducción significativa de la pancreatitis posterior a la CPRE comparada con el tratamiento secuencial de dos pasos CPRE más $\mathrm{CL}^{(6)}$.

La tasa de Ééxito terapeutico en la extracción de cálculos de la vía biliar entre el Rendez-vous y el tratamiento secuencial no reporta diferencia significativa ( $80 \%$ vs $95 \%)$, la incidencia de pancreatitis pos CPRE fue mayor en el grupo de tratamiento secuencial CPRE más CL. En cuanto al tratamiento en un solo paso entre el Rendez-vous y la ELVB no se reportaron diferencias significativas, excepto la no realización de la esfinterotomía $^{(1,2,3,4,5)}$.

En nuestro trabajo obtuvimos la canulación selectiva de la vía biliar con el trancístico en todos los pacientes (25) donde la CIO confirmo la presencia de coledocolitiasis en 14 pacientes, pudiendo ser realizado el paso de la guía en 12 pacientes lo que facilito ampliamente el procedimiento endoscópico en posición supina y así obteniendo una disminución significativa del tiempo de procedimiento.

Se obtuvo un $85,7 \%$ de éxito en la extracción de cálculos de la vía biliar con el método de Rendez-vous, encontrando como causa del $14,3 \%$ de fracaso a la presencia de cálculos que no permiten el paso de la guía. No hemos registrado episodios de pancreatitis post CPRE ni de otras complicaciones inherentes al procedimiento endoscópico.

En cuanto a la estadía hospitalaria pre y postoperatoria de nuestros pacientes fue reducida, con excepción de casos puntuales donde se realizaron el tratamiento en forma convencional y laparoscópica pura. La media de estadía hospitalaria fue de 5,8 días (2-23) y de estadía pos operatoria de 1, 2 días (1-8 días) similares a otras series e inclusive un poco menores debido a la selección de los pacientes $\left({ }^{1,2,3,4,5,9,10)}\right.$.

Las desventajas reportadas por los autores del método de Rendez-Vous están relacionadas a la infraestructura logística y de organización entre dos servicios, (Cirugía y Endoscopia), en

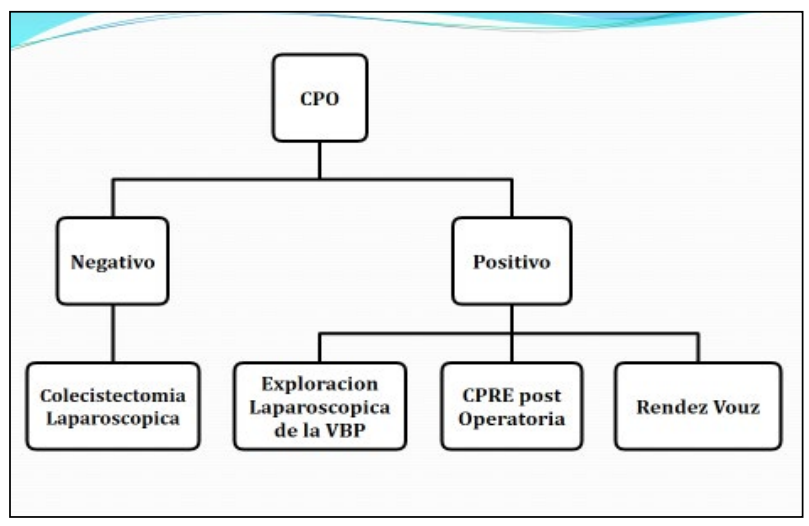

Figura 6. Algoritmo del manejo del paciente con sospecha intermedia de coledocolitiasis. Modificado. Hospital Nacional de Itauguá. Servicio de Cirugía General y Endoscopia Digestiva. 
nuestro trabajo no tuvimos dificultades debido a que el mismo equipo quirúrgico (cirujano, endoscopista) también es el mismo equipo que se encarga del servicio de Endoscopia Digestiva en nuestro hospital.

Tomando como referencia el diagrama o algoritmo de la ASGE en los pacientes con sospecha de coledocolitiasis proponemos que aquellos pacientes con riesgo intermedio, y no contando con estudios como la colangio resonancia o la Eco endoscopia, así como la ecografía laparoscópica, el método de Rendez-Vous es una buena opción terapéutica si se cuenta con todos los recursos humanos y la infraestructura para resolver la litiasis vesicular y coledociana en un solo tiempo. Es importante recalcar que el mejor tratamiento debe aplicarse de acuerdo a las situaciones de cada paciente, la disponibilidad de los recursos, el entrenamiento y experiencia en el manejo mínimo invasivo de las vias biliares ${ }^{(11)}$.

\section{REFERENCIAS BIBLIOGRÁFICAS}

1. Ahmed A ElGeidie, Gamal K ElEbidy, Yussef M Naeem. Esfinterotomía endoscópica preoperatoria versus intraoperatoria para el tratamiento de los cálculos del colédoco. Endoscopia quirúrgica, 2011 Abril, 25, 1230-1237

2. Rabago L, Collado D, Moran M. Chico I. Sa1490 ¿Cuál es el papel de la CPRE intraoperatoria en la actualidad y qué podemos esperar de ella? La experiencia de un hospital comunitario español Gastrointestinal Endoscopy 2011;04:186.

3. Bailey AA, Bourke MJ, Williams SJ, Walsh PR, Murray MA, Lee EY, Kwan V, Lynch PM. Un ensayo aleatorizado de la técnica de canulación en CPRE: efectos sobre el éxito técnico y de pancreatitis post-CPRE.Endoscopia. 2008; 40 : 296-301. PubMed

4. Fredrik S,Sara R,Lars E. La colangiopancreatografía retrógrada endoscópica con canulación cita reduce la lesión pancreática. Mundial J Gastroenterol. 28 de septiembre 2013; 19 (36): 6026 a 6034.

5. La Greca G, Barbagallo F, Sofia M, Latteri S, Russello D. encuentro laparoendoscópica simultánea para el tratamiento de la coledocolitiasis . Surg Endosc 2010; 24 : 769-780
6. Fredrik Swan, Sara Regner, Lars Enchsson. CPRE con canulación cita reduce la lesión pancreática. Mundial J Gastroenterology. 2013 Septiembre; 19(36) 6026-64.

7. Hong U, Xin I, Chen U. Comparison of laparoscopic cholecystectomy combined with intraoperative endoscopic sphinterotomy and laparoscopic exploration of the common bile duct for cholecystcholedocholithiasis. Surg. Endosc 2006; 20:424-7.

8. Diagnostico y manejo de la coledocolitiasis en la edad de oro de la imagen, endoscopia y laparoscopia. Mundial J Gastroenterol. 2014 octubre; 20(37): 382-401.

9. Jones M, Johnson M, Samourgian E. CPRE y colecistectomía laparoscpopica en un procedimiento combinado en comparación con tratamiento en dos pasos. Surg Endosc. 2013 Jun; 27(6): 1907-12.

10. ASGE Guideline. The role of endoscopy in the evaluación of suspected choledocholithiasis. Gastrointestinal Endoscopy 2010; 71 (1): 1-9.

11. Renato Costi AG, FDM y LS. Diagnóstico y manejo de la coledocolitiasis. World Journal of Gatroenterology. 2014 Octubre; 20(37). 